Short Research Communication

\title{
Chymase Activities and Survival in Endotoxin-Induced Human Chymase Transgenic Mice
}

Kazi Rafiq ${ }^{1 凶}$, Yu-Yan Fan ${ }^{1}$, Shamshad J. Sherajee ${ }^{1}$, Yoshimasa Takahashi², Junji Matsuura², Naoki Hase², Hirohito Mori ${ }^{3}$, Daisuke Nakano${ }^{1}$, Hideki Kobara ${ }^{3}$, Hirofumi Hitomi ${ }^{1}$, Tsutomu Masaki ${ }^{3}$, Hidenori Urata ${ }^{4}$, and Akira Nishiyama ${ }^{1}$

1. Department of Pharmacology, Kagawa University Medical School, Kagawa, Japan.

2. Teijin Institute for Bio-Medical Research, Teijin Pharma Ltd., Tokyo, Japan.

3. Department of Gastroenterology and Neurology, Kagawa University Medical School, Kagawa, Japan.

4. Department of Cardiovascular Diseases, Fukuoka University Chikushi Hospital, Fukuoka, Japan.

$\square$ Corresponding author: Kazi Rafiq, PhD, Department of Pharmacology, Kagawa University Medical School, 1750-1 Ikenobe, Miki-cho, Kita-gum, Kagawa 761-0793, Japan. Phone: +81 87891 2125; Fax: +81 87891 2126; E-mail: kazir73@med.kagawa-u.ac.jp or krafiq73@yahoo.com

(C) Ivyspring International Publisher. This is an open-access article distributed under the terms of the Creative Commons License (http://creativecommons.org/ licenses/by-nc-nd/3.0/). Reproduction is permitted for personal, noncommercial use, provided that the article is in whole, unmodified, and properly cited.

Received: 2013.08.12; Accepted: 2013.12.23; Published: 2014.01.II

\begin{abstract}
We examined the effects of overexpressed human chymase on survival and activity in lipopolysaccharide (LPS)-treated mice. Human chymase transgenic (Tg) and wild-type C57BL/6 (WT) mice were treated with LPS $(0.03,0.1$ and $0.3 \mathrm{mg} /$ day; intraperitoneal) for 2 weeks. Treatment with 0.03 $\mathrm{mg}$ LPS did not affect survival in either WT or Tg mice. WT mice were not affected by $0.1 \mathrm{mg} / \mathrm{day}$ of LPS, whereas $25 \%$ of $\mathrm{Tg}$ mice died. Survival of mice treated with $0.3 \mathrm{mg} /$ day of LPS was $87.5 \%$ and $0 \%$ in WT and Tg, respectively. LPS-induced increases in chymase activity in the heart and skin were significantly greater in $\mathrm{Tg}$ than WT mice. These data suggest a possible contribution of human chymase activation to LPS-induced mortality.
\end{abstract}

Key words: human chymase transgenic mice, chymase activity and lipopolysaccharide, endotoxemia

\section{Introduction}

Sepsis is a common problem in intensive care units, and is frequently complicated by the development of septic shock and multiorgan failure, reaching a mortality rate of nearly $50 \%$ [1]. Endotoxins such as lipopolysaccharide (LPS) released from gram-negative bacteria cause sepsis, which can be reproduced in experimental animals by the administration of LPS [2]. Sepsis is associated with cardiovascular dysfunction characterized by hypotension and decreased systemic vascular resistance, leading to high mortality rates [3], and the release of various inflammatory mediators [4]. The pathophysiological mechanisms responsible for LPS-induced cardiovascular dysfunction involve several mediators, including cytokines, angiotensin II, nitric oxide and catecholamine [5-7]. Suzuki et al. [8] showed that suffu- sion of LPS on the in situ hamster spinotrapezius muscle for $60 \mathrm{~min}$ elicits an immediate, reversible biphasic vasomotor response, vasoconstriction followed by vasodilatation, and increased accumulation of perivascular mast cells having chymase-like activity. Furthermore, both chymase inhibitors and $\mathrm{AT}_{1}$ receptor blockers abrogate LPS-induced responses [8], indicating a potential role for hamster chymase in the pathophysiology of septic shock. However, whether a similar role exists for human chymase has not been established. We sought to investigate the possible contribution of human chymase to LPS-induced mortality. Therefore, survival rate and chymase activity in the heart and skin were measured in transgenic mice carrying the human chymase gene [9]. 


\section{Materials and Methods}

\section{Animals}

All experimental procedures were performed according to the guidelines for the care and use of animals established by Kagawa University. The experiment was performed in male transgenic mice (18-20 weeks of age) carrying the human chymase gene (Tg) [9] and male wild-type C57BL/6 mice (WT). Preparation of the human chymase transgene and production of $\mathrm{Tg}$ mice were described previously in detail [9]. Mice were maintained in a pathogen-free facility under controlled temperature $\left(24 \pm 2^{\circ} \mathrm{C}\right)$ and humidity $(55 \pm 5 \%)$, with a 12 hrs light/dark cycle.

\section{Experimental Protocols and Other Analytical Procedures}

Basal chymase activity in the heart and skin $(n=6$, respectively) was measured in eighteen-week-old $\mathrm{Tg}$ and age-matched WT mice. Mice were sacrificed by an overdose of sodium pentobarbital (300 mg/ $\mathrm{kg}$, i.p.). The heart and approximately one square centimeter of skin tissue were removed and snap-frozen in liquid nitrogen and stored at $-80^{\circ} \mathrm{C}$. Chymase activity in the heart and skin was measured as described previously [9]. In the heart, mRNA levels of human chymase, mouse chymase (MMCP-4), angiotensin converting enzyme (ACE) and $\mathrm{AT}_{1 \mathrm{a}}$ receptor were also measured as described previously [10].

To examine the effect of LPS treatment, other 24 $\mathrm{Tg}$ mice at 18-20 weeks of age were divided in to three groups ( $n=8$ for each) and treated with intraperitoneal injection of LPS at $0.03,0.1$ or $0.3 \mathrm{mg} /$ day, respectively, for 2 weeks. Similarly, 24 age-matched WT mice were divided in to three groups ( $n=8$ for each) and treated the same. Although there is no previous report regarding the use of LPS in Tg mice, so according to the previous report [5] we used such modified doses. Heart and skin tissues were collected from surviving mice and chymase activity measured.

\section{Statistical Analysis}

Values are presented as the Mean \pm SD. Statistical comparisons were performed using one-way analysis of variance using a Bonferroni correction. Values of $P<0.05$ were considered statistically significant.

\section{Results}

Significant human chymase gene expression was observed in $\mathrm{Tg}$ heart, but not in WT, at baseline $(1.00 \pm 0.95$ and $2.31 \pm 1.40$ fold respectively, $P<0.05)$. In contrast, mRNA levels of MMCP-4, ACE, and $\mathrm{AT}_{1 a}$ receptor in heart were not different between $\mathrm{Tg}$ and WT mice (data not shown). As shown in Fig. 1, baseline chymase activity in the heart was similar between
WT and Tg mice, but was approximately 3-fold higher in Tg skin compared with WT skin. Baseline body weight was significantly lower in $\mathrm{Tg}$ mice compared with WT mice $(21.5 \pm 2.8$ and $25.7 \pm 2.6$ g respectively, $P<0.05)$. The proportion of mice surviving after LPS treatment was evaluated and the data analyzed by a Kaplan-Meier survival curve (Fig. 2). All WT mice $(100 \%)$ treated with 0.03 and $0.1 \mathrm{mg}$ LPS/day survived 14 days, with survival dropping slightly to $87.5 \%$ following treatment with $0.3 \mathrm{mg}$. In contrast, $\mathrm{Tg}$ mice treated with $0.3 \mathrm{mg}$ LPS/ day died within 4 days. Tg mice treated with 0.1 and $0.03 \mathrm{mg}$ LPS/day showed $75 \%$ and $100 \%$ survival, respectively. As shown in Fig. 3A, heart weight per body weight ratio, as an index of cardiac hypertrophy in LPS-treated Tg mice was significantly higher than that of LPS-treated WT mice. Chymase activity in the heart and skin was evaluated in mice treated with LPS (0.03 or $0.1 \mathrm{mg}$ ). Heart chymase activity was not significantly different between LPS-treated WT and Tg mice (Fig. 3B). As shown in Fig. 3C, skin chymase activity in LPS-treated $\mathrm{Tg}$ mice was approximately 3 -fold higher than that in LPS-treated WT mice, whose differences were also observed at baseline.

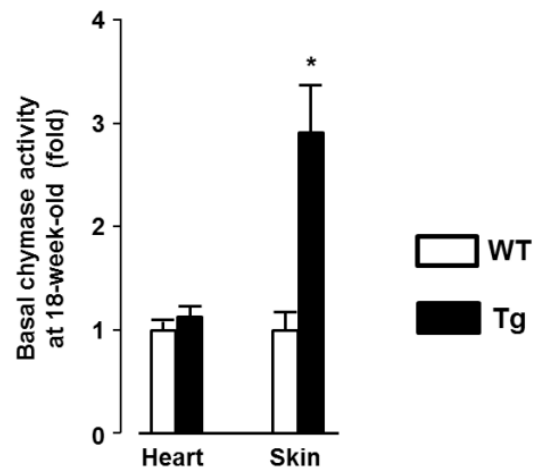

Figure I. Basal chymase activity in the heart and skin of 18-week-old wild-type C57BL/6 (WT) and transgenic ( $\mathrm{Tg}$ ) mice carrying the human chymase gene. Data are expressed as Mean \pm SD. $* P<0.05$.

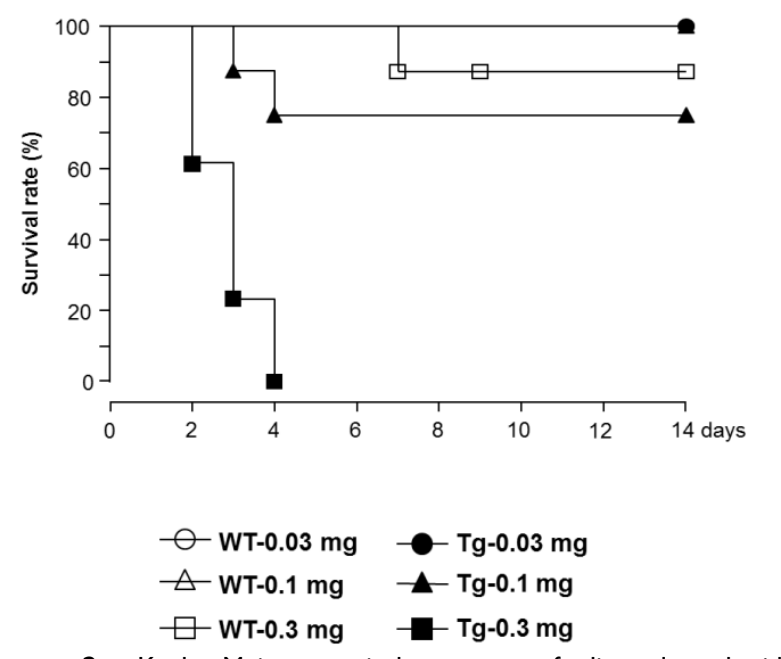

Figure 2. Kaplan-Meier survival curves of lipopolysaccharide (LPS)-treated WT and Tg mice. 
A

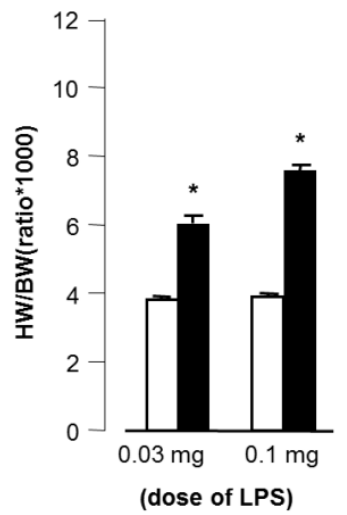

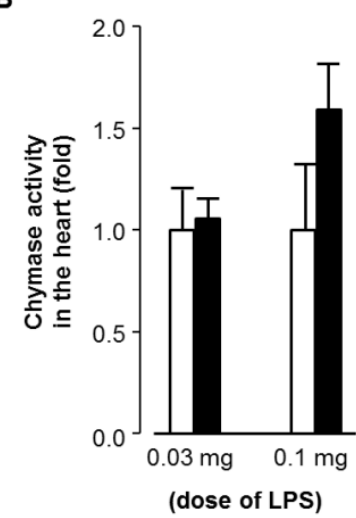

C

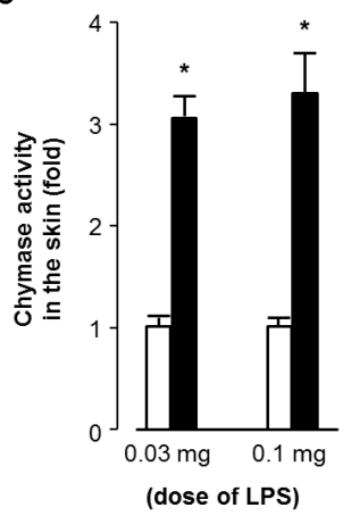

Figure 3. (A) Heart weight per body weight ratio of LPS-treated WT and Tg mice. (B) Chymase activity in the heart of LPS-treated WT and Tg mice. (C) Chymase activity in the skin of LPS-treated WT and Tg mice. HW; heart weight, BW; body weight. Data are expressed as Mean \pm SD. $* P<0.05$.

\section{Discussion}

At baseline, human chymase gene expression in heart was significantly higher but chymase activity is similar between WT and Tg mice. Treatment with LPS $\mathrm{Tg}$ mice showed cardiac hypertrophy, and heart chymase activity tended to show higher than in WT mice but these changes were not statistically significant. Previous studies showed that sepsis is associated with cardiovascular dysfunction [3]. We therefore speculate that LPS-induced cardiovascular dysfunction may be augmented, at least in part, by over-expression of the human chymase gene. This concept is consistent with a recent study by Koga et al. [9] that showed human chymase expression in mice induces mild hypertension with left ventricular hypertrophy, characterized by cardiomyocyte hyperplasia and increased fibrosis in the left ventricle. From this data it is difficult to explain about the role of human chymase in LPS-induced increase mortality is due to cardiovascular dysfunction. Because of the low prevalence of survival of LPS induced $\mathrm{Tg}$ mice, we did not evaluate any cardiac functional parameters. Significant human chymase gene expression was observed in heart of $\mathrm{Tg}$ mice, however, chymase activity was not upregulated. From these preliminary data it is difficult to explain why chymase activity was not significantly upregulated in the hearts of $\mathrm{Tg}$ mice. Further studies are needed to investigate the role of chymase to the development of LPS-induced cardiac or renal injury. Therefore, studies will be definitely emergence to link chymase activity with these observations.

We observed that $\mathrm{Tg}$ mice have phenotypic characteristic of alopecia, consistent with previous studies $[9,11]$. However, the mechanisms responsible for alopecia in $\mathrm{Tg}$ mice remained unclear. Clinical studies have shown that mast cells are involved in the pathogenesis of various types of hair loss and alopecia. In scarring alopecia $[12,13]$ and androgenetic alopecia [14], the number of skin mast cells is remarkably increased. An increase in mast cells is potentially one of the reasons for remarkably higher chymase activity in skin than other organs, which may lead to alopecia in $\mathrm{Tg}$ mice. This hypothesis is supported by the data in which $\mathrm{Tg}$ mice exhibited higher levels of chymase activity in the skin. We also observed similar elevated skin chymase activity between LPS-treated and non-treated Tg mice. However, the pathophysiological impact on the role of skin chymase in LPS-induced mortality remains unknown.

In conclusion, we demonstrate that human chymase transgenic mice elicit a markedly lower survival in response to LPS treatment. These data suggest that human chymase contributes to mortality through endotoxemia. However, further studies are needed to determine the precise mechanisms by which human chymase contributes to cardiovascular or renal dysfunction during endotoxemia.

\section{Acknowledgements}

This work was supported in part by a Grant-in-Aid for Scientific Research from the Ministry of Education, Culture, Sports, Science and Technology of Japan (to Akira Nishiyama).

\section{Competing Interests}

The authors have declared that no competing interest exists. 


\section{References}

1. Rangel-Frausto MS, Pittet D, Costigan M, Hwang T, Davis CS, Wenzel RP. The natural history of the systemic inflammatory response syndrome (SIRS). A prospective study. Jama. 1995;273(2):117-23.

2. Haeffner-Cavaillon N, Carreno MP, Aussel L, Caroff M. Molecular aspects of endotoxins relevant to their biological functions. Nephrol Dial Transplant. 1999;14(4):853-60.

3. Parrilo JE. Pathogenic mechanisms of septic shock. New England Journal of Medicine 1993;328:1471-7.

4. Mehanna A, Vitorino DC, Panis C, Blanco EE, Pinge-Filho P, Martins-Pinge MC. Cardiovascular and pulmonary effects of NOS inhibition in endotoxemic conscious rats subjected to swimming training. Life Sci. 2007;81(16):1301-8.

5. Niimi R, Nakamura A, Yanagawa Y. Suppression of endotoxin-induced renal tumor necrosis factor-alpha and interleukin-6 mRNA by renin-angiotensin system inhibitors. Jpn J Pharmacol. 2002;88(2):139-45.

6. Henrich WL, Hamasaki Y, Said SI, Campbell WB, Cronin RE. Dissociation of systemic and renal effects in endotoxemia. Prostaglandin inhibition uncovers an important role of renal nerves. J Clin Invest. 1982;69(3):691-9.

7. Schaller MD, Waeber B, Nussberger J, Brunner HR. Angiotensin II, vasopressin, and sympathetic activity in conscious rats with endotoxemia. Am J Physiol. 1985;249(6 Pt 2):H1086-92.

8. Suzuki H, Caughey GH, Gao XP, Rubinstein I. Mast cell chymase-like protease(s) modulates Escherichia coli lipopolysaccharide-induced vasomotor dysfunction in skeletal muscle in vivo. J Pharmacol Exp Ther. 1998;284(3):1156-64

9. Koga $\mathrm{T}$, Urata $\mathrm{H}$, Inoue $\mathrm{Y}$, et al. Human chymase expression in a mice induces mild hypertension with left ventricular hypertrophy. Hypertens Res. 2003;26(9):759-68.

10. Wei CC, Hase $\mathrm{N}$, Inoue $\mathrm{Y}$, et al. Mast cell chymase limits the cardiac efficacy of Ang I-converting enzyme inhibitor therapy in rodents. J Clin Invest. 2010;120(4):1229-39.

11. Rafiq K, Sherajee SJ, Fan YY, et al. Blood glucose level and survival in streptozotocin-treated human chymase transgenic mice. Chin J Physiol. 2011;54(1):30-5.

12. Reed RJ, Clark WH, Mihm MC. The cutaneous mucinoses. Hum Pathol. 1973;4(2):201-5.

13. Rook AH, Prystowsky MB, Cassin M, Boufal M, Lessin SR. Combined therapy for Sezary syndrome with extracorporeal photochemotherapy and low-dose interferon alfa therapy. Clinical, molecular, and immunologic observations. Arch Dermatol. 1991;127(10):1535-40.

14. Lattanand A, Johnson WC. Male pattern alopecia a histopathologic and histochemical study. J Cutan Pathol. 1975;2(2):58-70. 